\title{
A Procedure to Estimate the Size of a Suction Flap for a Passive HLFC System
}

\author{
Geza Schrauf \\ DLR, Lilienthalplatz 7, 38108 Braunschweig, Germany \\ geza.schrauf@dlr.de
}

\begin{abstract}
We describe a simple pre-design method to estimate the size of an air outlet flap for a passive hybrid laminar flow control (HLFC) system to determine the feasibility of that flap. The method is based on an ingenious application of old experimental results. The computational effort is minimal.
\end{abstract}

Keywords: Hybrid Laminar Flow Control (HLFC), passive suction system, feasibility of passive suction, size of air outlet, suction flap

\section{Nomenclature}

$A_{\text {in }}$ Inflow cross-section area of the duct to the outlet $\left[\mathrm{m}^{2}\right]$.

$A_{\text {out }}$ Outflow cross-section area of the outlet $\left[\mathrm{m}^{2}\right]$.

$C_{\text {dis }}$ Discharge coefficient as defined on page 6.

$\dot{m}=\dot{m}_{\text {in }}=\dot{m}_{\text {out }}$. Mass flow through inflow or outflow cross-section $[\mathrm{kg} / \mathrm{s}]$.

$p_{\text {in }} \quad$ Static pressure at the inflow cross-section $[\mathrm{Pa}]$.

$p_{\text {out }}$ Static pressure at the outflow cross-section $[\mathrm{Pa}]$.

$p_{\text {ref }}$ Reference pressure $[\mathrm{Pa}]$.

$\bar{v}_{i n} \quad$ Mean velocity in inflow cross-section $\bar{v}_{i n}=\dot{m}_{i n} /\left(\rho A_{i n}\right)[\mathrm{m} / \mathrm{s}]$.

$\bar{v}_{\text {out }}$ Mean velocity in outflow cross-section $\bar{v}_{\text {out }}=\dot{m}_{\text {out }} /\left(\rho A_{\text {out }}\right)[\mathrm{m} / \mathrm{s}]$.

$v_{\text {ref }}$ Reference velocity $[\mathrm{m} / \mathrm{s}]$.

$\dot{V}_{\text {in }}$ Volume flow through the inflow cross-section $\left[\mathrm{m}^{3} / \mathrm{s}\right]$.

$\dot{V}_{\text {out }}$ Volume flow through the outflow cross-section $\left[\mathrm{m}^{3} / \mathrm{s}\right]$.

$\delta \quad$ Flap opening angle [degree].

$\rho \quad$ Density $\left[\mathrm{kg} / \mathrm{m}^{3}\right]$.

$q_{*} \quad$ Subscript '*' denotes a non-dimensional quantity.

\section{Introduction}

In this paper we present a simple method for estimating the cross-section area, and with that, the size of an outlet that discharges air from an internal flow system into the external flow around an aircraft. The method is an ingenious application of wind tunnel results from 1938 [1]. It might prove very useful for feasibility and pre-design studies of passive HLFC systems. 


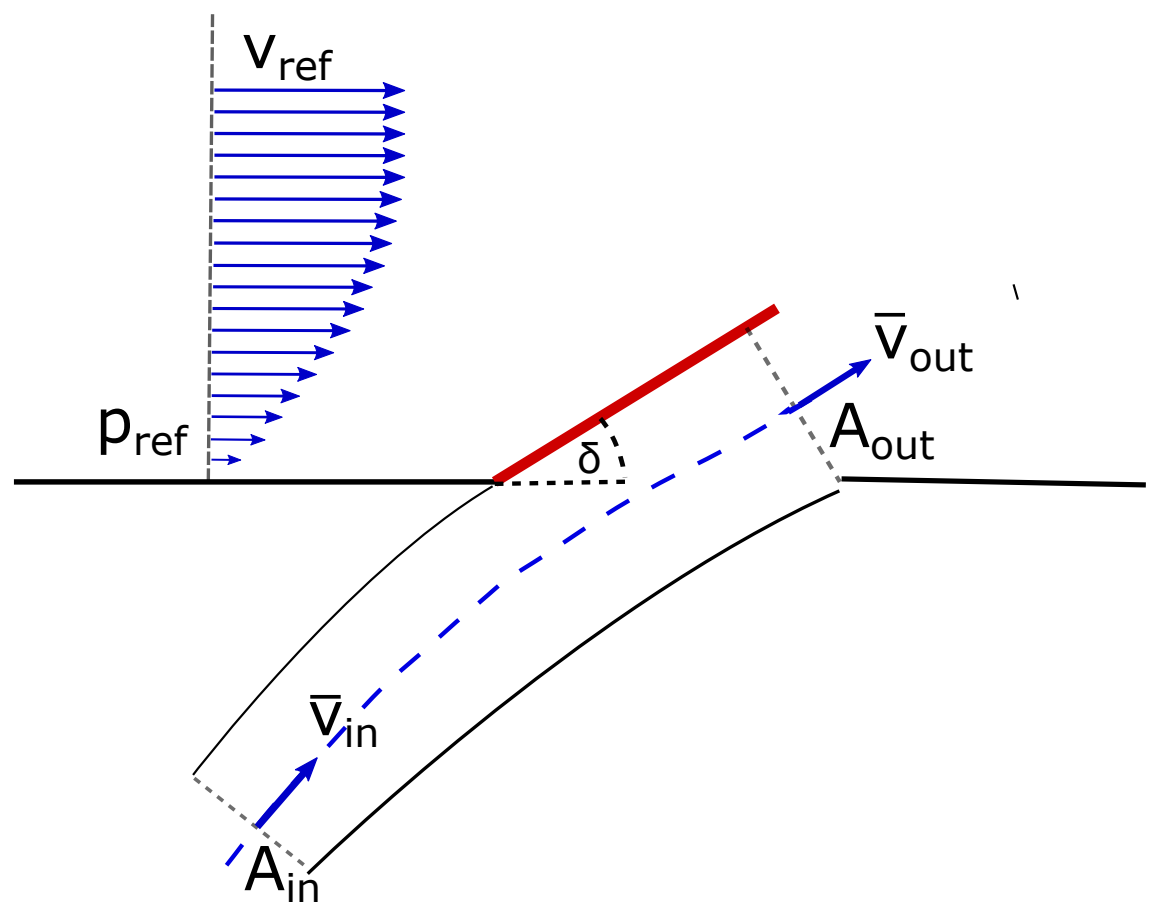

Fig. 1. Sketch of a flap outlet.

We describe the method for a simple flap outlet. However, analogous estimates can be derived for outlets with different shapes, such as circular holes or "Lamellen"-outlets.

\section{Review of Rogallo's Results}

Figure 1 shows a sketch of a simple flap outlet. The inflow part of the outlet is a tube ${ }^{1}$ with cross-section area $A_{\text {in }}$. By opening the flap, we can observe an outflow. The cross-section area $A_{\text {out }}$ of an outlet is usually defined as the minimal cross-section area, i.e. for a planar flap, it is measured perpendicular to its surface.

Quantities of the external flow, somewhat upstream of the outlet, are taken as reference quantities for the internal flow. We assume that, on the one hand, this location is close enough to the outlet so that the reference pressure $p_{r e f}$ at this location is equal to the surface (or static) pressure at the outlet when the flap is closed. On the other hand, it is assumed that the location is far enough upstream of the outlet so that the reference pressure is not changed when the outlet is opened. This is, of course, an idealization. As reference velocity $v_{r e f}$ we

\footnotetext{
${ }^{1}$ The shape of the inflow duct is irrelevant, that is the inflow duct can also be a rectangular channel, as, for example, used for the outlets in [1, see p. 5].
} 


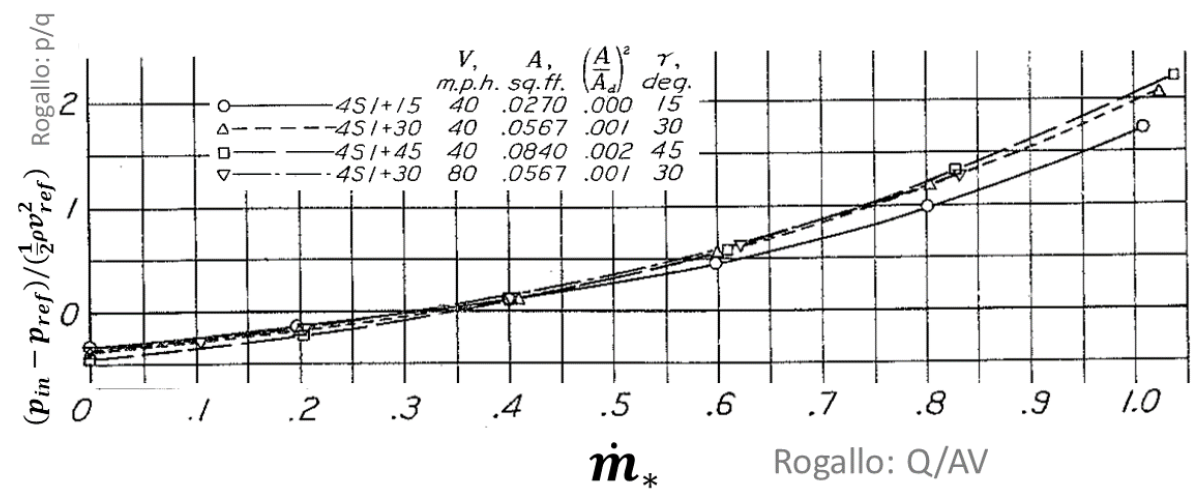

Fig. 2. Part of Figure 43 from [1] with our and Rogallo's notation.

take the velocity at the edge of the boundary layer at this location, i.e., somewhat upstream of the flap ${ }^{2}$. Again, we assume that this velocity is not influenced by the opening of the flap.

The input data required for our estimate are the reference pressure $p_{r e f}$, the reference velocity $v_{r e f}$, as well as the inflow static pressure $p_{i n}$ and the mass flow $\dot{m}_{i n}$ through the inflow cross-section area.

Based on the reference velocity $v_{r e f}$, we define the non-dimensional mass flow through the outlet as

$$
\dot{m}_{*}=\frac{\dot{m}_{\text {out }}}{\rho A_{\text {out }} v_{\text {ref }}}
$$

For incompressible flow, we can consider the non-dimensional volume flow

$$
\dot{V}_{*}=\frac{\dot{V}_{\text {out }}}{A_{\text {out }} v_{\text {ref }}}=\frac{\bar{v}_{\text {out }}}{v_{\text {ref }}},
$$

where $\bar{v}_{\text {out }}$ is the mean velocity through the outflow area.

To make the input pressure $p_{\text {in }}$ non-dimensional, Rogallo defines the pressure coefficient $\left(p_{\text {in }}-p_{\text {ref }}\right) /\left(1 / 2 \rho v_{r e f}^{2}\right)$ based on the above reference quantities. He then presents the results of his wind tunnel measurements for each specific outlet configuration in the form of

$$
\frac{p_{\text {in }}-p_{\text {ref }}}{1 / 2 \rho v_{\text {ref }}^{2}}=f\left(\dot{V}_{*}\right),
$$

\footnotetext{
${ }^{2}$ This definition is similar to the reference velocity we use to describe the influence of forward and backward facing steps on laminar flow. Similar to our case, we use as reference the velocity at the boundary layer edge at the location of the step, however, for a smooth surface without the step.
} 
describing the variation of the pressure coefficient with the non-dimensional volume flow $\dot{V}_{*}$. In [1] we find plots of such relations for many different types of outlets. Those plots are reproduced in [2]. In Figure 43 of [1], Rogallo presents the results of his wind tunnel measurements for a simple flap with the three opening angles of $15^{\circ}, 30^{\circ}$, and $45^{\circ}$ degree. In our Figure 2, we reproduce a part of his figure with our notation for the $\mathrm{x}$ - and $\mathrm{y}$-axes. We see that the functions are more or less independent of the opening angle and the flow velocity. From this figure we extract the following numerical values on which we will base our approximation method:

\begin{tabular}{c|c|c|c|c} 
& $\begin{array}{c}15^{\circ} \\
40 \text { m.p.h. } .\end{array}$ & $\begin{array}{c}30^{\circ} \\
40 \text { m.p.h. }\end{array}$ & $\begin{array}{c}45^{\circ} \\
40 \text { m.p.h. }\end{array}$ & $\begin{array}{c}30^{\circ} \\
\text { m.p.h. } .\end{array}$ \\
\hline$Q / A V$ & $p / q$ & $p / q$ & $p / q$ & $p / q$ \\
\hline 0.0 & $-0,32$ & $-0,35$ & $-0,43$ & $-0,36$ \\
0.2 & $-0,16$ & $-0,17$ & $-0,21$ & $-0,18$ \\
0.4 & 0,11 & 0,12 & 0,11 & 0,14 \\
0.6 & 0,47 & 0,52 & 0,55 & 0,57 \\
0.8 & 0,98 & 1,10 & 1,20 & 1,20 \\
1.0 & 1,60 & 1,80 & 1,90 &
\end{tabular}

Table 1. Numerical values taken from [1, Fig. 43].

Rogallo's " $p / q$ " is in our notation $\left(p_{\text {in }}-p_{\text {ref }}\right) /\left(1 / 2 \rho v_{r e f}^{2}\right)$ and his " $Q / A V$ " corresponds to our $\dot{m}_{\star}$. The first line of the table contains the pressure values for zero mass flow. We find that the pressure coefficient is not zero but has an approximate value of -0.4 . This means that a flap outlet can produce some underpressure, i.e., that it has a suction capability. This is necessary for a passive HLFC system. Not every outlet has this property, as can be seen, for example, from Figure 25 of [2] for a circular-hole outlet ${ }^{3}$.

For zero mass flow, the static pressure $p_{\text {out }}$ in the outflow cross-section is equal to the pressure $p_{i n}$, as can be seen from the inviscid Bernoulli equation (6). Using $p_{\text {out }}=p_{\text {in }}$, we obtain for zero mass flow

$$
\frac{p_{\text {out }}-p_{\text {ref }}}{1 / 2 \rho v_{\text {ref }}^{2}} \approx-0.4
$$

With this relation, we can estimate the static pressure $p_{\text {out }}$ in the outflow crosssection of a flap opening as

$$
p_{\text {out }}=p_{\text {ref }}-0.4 \cdot 1 / 2 \rho v_{\text {ref }}^{2} .
$$

To derive our estimates, we assume that this relation for the static pressure $p_{\text {out }}$ is also valid for non-zero mass flow.

\footnotetext{
${ }^{3}$ For a circular-hole outlet we obtain from [2, Figure 25] that $p_{i n}=p_{r e f}$, i.e. a circular hole has no suction power.
} 


\section{Assumption A:}

The static pressure $p_{\text {out }}$ is independent of the mass (or volume) flow through the outlet and can be approximated by (5).

This assumption seems to be a good first approximation. To support this, we present values obtained from a $3 \mathrm{D}$ Navier-Stokes calculation for a simple

\begin{tabular}{c|c|c||c|c}
$\delta$ & $p_{\text {in }}$ & $p_{\text {out }, N S}$ & $p_{\text {out }}$ & Error \\
{$[$ deg $]$} & {$[$ Pa $]$} & {$[P a]$} & {$[$ Pa $]$} & {$[\%]$} \\
\hline $7.5^{\circ}$ & 21879 & 19715 & 19329 & $2.0 \%$ \\
$10^{\circ}$ & 21877 & 19467 & 19329 & $0.7 \%$ \\
\hline $10^{\circ}$ & 20878 & 19496 & 19329 & $0.9 \%$ \\
$10^{\circ}$ & 21877 & 19497 & 19329 & $0.7 \%$ \\
$10^{\circ}$ & 22876 & 19496 & 19329 & $0.9 \%$ \\
$10^{\circ}$ & 23875 & 19337 & 19329 & $0.0 \%$
\end{tabular}

Table 2. Comparison of computed and estimated values of $p_{\text {out }}$.

flap outlet with an aspect ratio ${ }^{4}$ of 2.6 for a case with $M_{\infty}=0.85$. We see that the pressure $p_{\text {out }}$ obtained with the approximation (5) is close to the computed values $p_{\text {out }, N S}$, which are obtained as averages over $A_{\text {out }}$. Furthermore, the example shows that the computed pressure $p_{\text {out }, N S}$ does not change much when the opening angle $\delta$ or the pressure $p_{i n}$ in the inflow cross-section are varied.

\section{Approximation of the Experimental Results}

In this section we show how to use the values of Table 1 to derive the main relation for our approximation method. We assume the flow between $A_{\text {in }}$ and $A_{\text {out }}$ to be incompressible and inviscid, so that we can apply the Bernoulli equation (6). With this equation we obtain the ideal mean outflow velocity with the help of the inflow pressure $p_{i n}$, the mean inflow velocity $\bar{v}_{i n}$, and the static pressure $p_{\text {out }}$ as

$$
\frac{1}{2} \rho \bar{v}_{\text {out_ideal }}^{2}=p_{\text {in }}+\frac{1}{2} \rho \bar{v}_{\text {in }}^{2}-p_{\text {out }},
$$

or

$$
\bar{v}_{\text {out_ideal }}=\sqrt{\frac{2}{\rho}\left[p_{\text {in }}+\frac{1}{2} \rho \bar{v}_{\text {in }}^{2}-p_{\text {out }}\right]} .
$$

\footnotetext{
${ }^{4}$ The aspect ratio of a simple flap is defined as the ratio of spanwise to chordwise extent.
} 
The ideal mass flow through the outflow cross-section is

$$
\dot{m}_{\text {out_ideal }}=\rho \bar{v}_{\text {out_ideal }} A_{\text {out }} .
$$

Due to the effect of viscosity (and local flow separation) the real mass flow $\dot{m}_{\text {out }}$ through $A_{\text {out }}$ will be smaller. Therefore, we define a discharge coefficient ${ }^{5}$ as follows:

$$
C_{\text {dis }}=\frac{\dot{m}_{\text {out }}}{\dot{m}_{\text {out_ideal }}}=\frac{\rho A_{\text {out }} \bar{v}_{\text {out }}}{\rho A_{\text {out }} \bar{v}_{\text {out_ideal }}} .
$$

For incompressible flow through an orifice, the discharge coefficient is reduced to the velocity ratio

$$
C_{\text {dis }}=\frac{\bar{v}_{\text {out }}}{\bar{v}_{\text {out_ideal }}} .
$$

With equation (7) and $\bar{v}_{\text {in }} A_{\text {in }}=\bar{v}_{\text {out }} A_{\text {out }}$, we obtain

$$
C_{\text {dis }}=\frac{\bar{v}_{\text {out }} / v_{\text {ref }}}{\bar{v}_{\text {out_ideal }} / v_{\text {ref }}}=\frac{\frac{\bar{v}_{\text {out }}}{v_{\text {ref }}}}{\sqrt{\frac{p_{\text {in }}-p_{\text {out }}}{\frac{1}{2} \rho v_{\text {ref }}^{2}}+\left(\frac{\bar{v}_{\text {out }}}{v_{\text {ref }}}\right)^{2}\left(\frac{A_{\text {out }}}{A_{\text {in }}}\right)^{2}}} .
$$

Our $p_{i n}$ corresponds to Rogallo's duct pressure $p_{d}$ and we can write (11) as

$$
C_{d i s}=\frac{\frac{\bar{v}_{\text {out }}}{v_{\text {ref }}}}{\sqrt{\frac{p_{d}-p_{\text {ref }}}{\frac{1}{2} \rho v_{\text {ref }}^{2}}-\frac{p_{\text {out }}-p_{\text {ref }}}{\frac{1}{2} \rho v_{\text {ref }}^{2}}+\left(\frac{\bar{v}_{\text {out }}}{v_{\text {ref }}}\right)^{2}\left(\frac{A_{\text {out }}}{A_{d}}\right)^{2}}} .
$$

According to our main assumption, the static pressure $p_{\text {out }}$ in the outlet crosssection can be approximated by (5), so that the second term in the denominator becomes -0.4 . The first term $\left(p_{d}-p_{\text {ref }}\right)\left(\frac{1}{2} \rho v_{r e f}^{2}\right)$ is the " $p / q$ "-value listed in Table 1. This allows us to compute the discharge coefficients directly from the tabulated values for each flap setting.

In the next step we consider the non-dimensional mass flow

$$
\dot{m}_{*}=\frac{\dot{m}_{\text {out }}}{\rho v_{\text {ref }} A_{\text {out }}}=\frac{\bar{v}_{\text {out }}}{v_{\text {ref }}}
$$

\footnotetext{
${ }^{5}$ The discharge coefficient is a mass flow ratio and must be distinguished from the contraction number or contraction ratio which is defined as an area ratio. For incompressible flow, the discharge coefficient becomes a volume flow ratio. When considering the incompressible flow through only one opening, it is then reduced to a ratio of area-averaged velocities. We point out that the discharge coefficient is not well-defined for very small mass flows. For $\dot{m}_{\text {out }} \rightarrow 0$, we obtain an expression of the type $" 0 / 0 "$.
} 


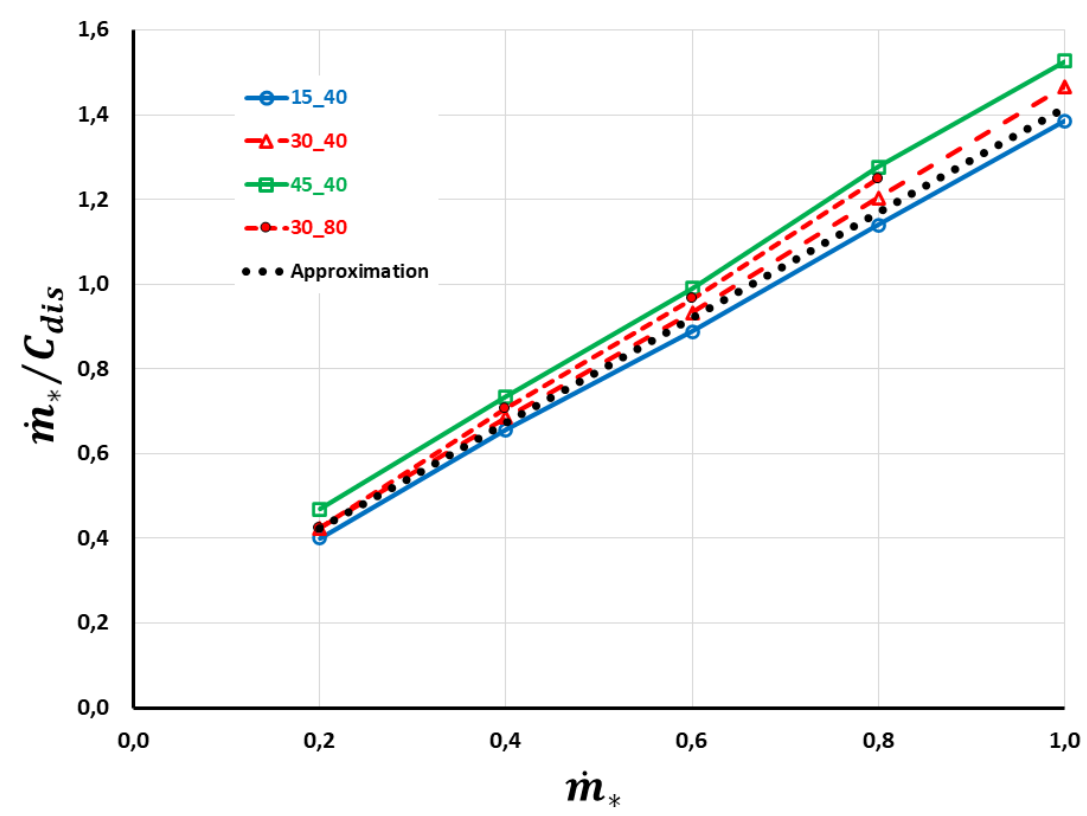

Fig. 3. Plot of the functions $f_{\delta}$.

and re-plot Figure 2 in the form ${ }^{6}$

$$
\frac{\dot{m}_{*}}{C_{d i s}}=f_{\delta}\left(\dot{m}_{*}\right)
$$

The result is shown in Figure 3. We see that each flap setting is represented by a nearly linear relation

$$
\frac{\dot{m}_{*}}{C_{d i s}}=f_{\delta}\left(\dot{m}_{*}\right)=a(\delta) \dot{m}_{*}+b(\delta),
$$

with coefficients $a(\delta)$ and $b(\delta)$ that can be approximated by

$$
\begin{aligned}
& a(\delta)=0.0028 \delta+1.18 \\
& b(\delta)=0.0016 \delta+0.14 .
\end{aligned}
$$

In Figure 3, we include an additional line to illustrate the approximation for a flap angle of $22^{\circ}$.

\footnotetext{
${ }^{6}$ Initially, we tried to approximate the functions $C_{d i s}=\tilde{f}_{\delta}\left(\dot{m}_{*}\right)$ with polynomials or elliptic curves. However, working with the functions $f_{\delta}$ of the formulation (14) is easier and leads to more accurate estimates, because the functions $f_{\delta}$ are nearly linear.
} 


\section{Flap Size Estimation}

Now we want to estimate the cross-section area for an outlet flap which is supposed to discharge a certain mass flow $\dot{m}$ for an inlet pressure $p_{i n}$. The mass flow leaving the flap through its outflow cross-section $A_{\text {out }}$ is equal to the mass flow entering through $A_{i n}$. Therefore, we have $\bar{v}_{i n}=\dot{m} /\left(\rho A_{i n}\right)$, and with that, we know the quantities $p_{r e f}, v_{r e f}, \rho, p_{i n}, \bar{v}_{i n}$. Our estimation procedure consists of two steps:

\section{Step 1:}

With the reference quantities and density $\rho$ we compute $p_{\text {out }}$ with formula (5). To generate an outflow, the outlet pressure $p_{\text {out }}$ must be smaller than the inlet pressure $^{7} p_{\text {in }}$, i.e.

$$
p_{\text {out }}<p_{\text {in }}
$$

If this condition is violated, an HLFC system with the plenum pressure $p_{p}=p_{\text {in }}$ cannot operate in passive mode, and we need to redesign to allow for a higher plenum pressure.

Step 2:

Using formulas (7), (10) and (13) we obtain

$$
\frac{\dot{m}_{*}}{C_{\text {dis }}}=\frac{1}{v_{\text {ref }}} \sqrt{\frac{2}{\rho}\left[p_{\text {in }}+\frac{1}{2} \rho \bar{v}_{\text {in }}^{2}-p_{\text {out }}\right]},
$$

and with (15) we get

$$
a(\delta) \dot{m}_{*}+b(\delta)=\frac{1}{v_{\text {ref }}} \sqrt{\frac{2}{\rho}\left[p_{\text {in }}+\frac{1}{2} \rho \bar{v}_{\text {in }}^{2}-p_{\text {out }}\right]} .
$$

This formula allows us to compute the non-dimensional mass flow $\dot{m}_{*}$ for a given flap angle $\delta$. This non-dimensional mass flow $\dot{m}_{*}$ is the main input for our estimations. Because of

$$
\dot{m}_{*}=\frac{\dot{m}_{\text {out }}}{\rho v_{\text {ref }} A_{\text {out }}}=\frac{\dot{m}_{\text {in }}}{\rho v_{\text {ref }} A_{\text {out }}},
$$

we can either obtain the outflow cross-section area for a given mass flow $\dot{m}_{i n}$ as

$$
A_{\text {out }}=\frac{\dot{m}_{\text {in }}}{\rho v_{\text {ref }} \dot{m}_{*}},
$$

or the mass flow for a given outflow area $A_{\text {out }}$ (defined by the opening angle $\delta$ ) with the help of

$$
\dot{m}=\dot{m}_{*} \rho v_{\text {ref }} A_{\text {out }} .
$$

\footnotetext{
${ }^{7}$ If $\Delta p$ is the pressure loss from the plenum to the inlet cross-section, then $p_{\text {out }}$ must be smaller than $p_{i n}=p_{p}-\Delta p$.
} 
The area obtained with formula (22) can be used to assess the feasibility of a passive HLFC system: if the area is too large, then the envisaged passive HLFC system with plenum pressure $p_{p}$ and mass flow $\dot{m}$ is not feasible. Furthermore, with (23), we can estimate the suction capability of a simple flap when installed at a certain location.

The quantity $\dot{m}_{\star}$ is the basis for our estimation. Therefore, in Table 3, we present values of $\dot{m}_{\star}$ estimated with (20) and compare them to values $\dot{m}_{\star, N S}$ obtained from a 3D Navier-Stokes calculations. The computational cases are the

\begin{tabular}{c|c|c||c|c}
$\delta$ & $p_{i n}$ & $\dot{m}_{\star, N S}$ & $\dot{m}_{\star}$ & Error \\
{$[$ deg] } & {$[P a]$} & {$[-]$} & {$[-]$} & {$[\%]$} \\
\hline $7.5^{\circ}$ & 21879 & 0.259 & 0.243 & $-6.4 \%$ \\
$10^{\circ}$ & 21877 & 0.266 & 0.240 & $-9.9 \%$ \\
\hline $10^{\circ}$ & 20878 & 0.204 & 0.157 & $-23 \%$ \\
$10^{\circ}$ & 21877 & 0.266 & 0.240 & $-9.9 \%$ \\
$10^{\circ}$ & 22876 & 0.318 & 0.308 & $-3.2 \%$ \\
$10^{\circ}$ & 23875 & 0.367 & 0.367 & $0.0 \%$
\end{tabular}

Table 3. Comparison of computed and estimated values of $\dot{m}_{\star}$.

same as in Table 2. From this table, we see that we have a reasonable agreement. Only the case $\delta=10^{\circ}$ and $p_{i n}=20878 \mathrm{~Pa}$, i.e. the one with the weakest suction, exhibits a large error. This might be caused by the three-dimensional flow around the flap sides, which becomes dominant when the outflow is weak. In all other cases considered, including flaps with smaller aspect ratios or at different locations, the error in $\dot{m}_{\star}$ remained below $12 \%$.

\section{Conclusions}

We present a method to estimate the size of a suction flap for a passive HLFC system. Such an estimate proves useful during the pre-design phase, when we need to find out whether an envisaged flap would be as small as a matchbox or as large as a barn door. If the estimated size is too large for the envisaged location, then we can drop this flap design without performing further calculations with expensive methods such as Navier-Stokes calculation. This saves a lot of time and effort.

\section{References}

1. Rogallo, Francis M., Gauvain, William E., "Wind-Tunnel Investigation of Air Inlet and Air Outlet Openinge for Aicraft." NACA Special Report No. 95, 1938.

2. Rogallo, F. M., "Internal Flow Systems for Aircraft." NACA Report No. 713, 1941. 